\title{
A typical atypical pneumonia
}

\author{
J Mikhael MD ${ }^{1}$, S Humphrey MD FRCPC ${ }^{1}$, M Jabi MD FRCPC ${ }^{2}$, AD Badley MD FRCPC ${ }^{1,3}$
}

\begin{abstract}
A 55-year-old white man from eastern Ontario with a past medical history of class IV angina and no previous significant infectious disease history presented to the emergency room with a five-day history of left-sided, pleuritic chest pain, dyspnea and cough productive of clear sputum. He had no hemoptysis, chills or previous exposure to tuberculosis, but had lost $9 \mathrm{~kg}$ in the previous month. He had not travelled recently, was not exposed to any unusual animals and had no pets. He smoked 40 packs of cigarettes/year. His medications were acetylsalicylic acid, nitroglycerine and metoprolol.

On admission, he was febrile and hypoxic (oxygen pressure of 69 breathing room air), yet his chest was clear to auscultation. His white blood cell count was $10.5 \times 10^{9} / \mathrm{L}$, with a normal differential, and the sedimentation rate was $215 \mathrm{~mm} / \mathrm{h}$. Chest radiography showed a lateral, diamondshaped area of opacity in the left mid-lung zone, abutting the chest wall (Figure 1). A D-dimer assay was negative and a ventilation perfusion scan was interpreted as being of low probability for pulmonary embolism. The patient was treated with intravenous erythromycin and cefuroxime, but remained both febrile and hypoxic. HIV serology and sputum cultures were negative. A biopsy of the lesion was performed.
\end{abstract}

What is your diagnosis?

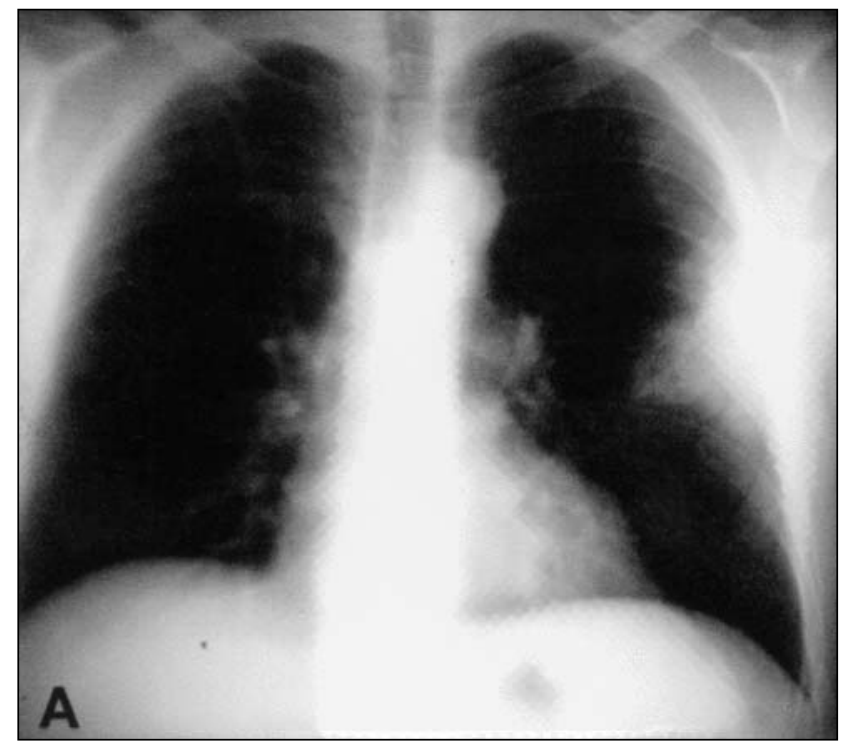

Figure 1) Chest radiography before therapy. X-ray reveals a diamond-shaped opacity in the left mid-lung zone

Departments of ${ }^{1}$ Medicine, ${ }^{2}$ Pathology and ${ }^{3}$ Division of Infectious Diseases, Ottawa Hospital, Ottawa, Ontario

Correspondence and reprints: Dr Andrew Badley, Ottawa Hospital, General Campus, 501 Smyth Road, Ottawa, Ontario K1H 8L6.

Telephone 613-737-8998,fax 613-737-8682,e-mail abadley@ohri.ca 


\section{CLINICAL VIGNETTE}

continued from page 20

\section{DIAGNOSIS}

An ultrasound-guided biopsy of the lesion revealed granulomatous inflammation with encapsulated fungal spores, which was suggestive of cryptococcosis. Silver and mucin stains revealed fungal elements (Figure 2). Fungal cultures confirmed the presence of Cryptococcus neoformans. The patient was treated with daily oral doses of fluconazole (400 $\mathrm{mg}$ ) and showed clinical improvement within one month. Fluconazole therapy was stopped after six months and a follow-up chest x-ray confirmed complete resolution (Figure 3).

\section{DISCUSSION}

The patient presented with a case of pulmonary Cryptococcus neoformans. The patient was HIV-negative and had no history that suggested an underlying immune deficiency. He presented to the emergency room with fever, cough and an atypical pulmonary infiltrate. The differential diagnosis included malignancy, mycobacterial infection, fungal infection and bacterial pneumonia. Following biopsy, which confirmed a cryptococcus infection, treatment with fluconazole was successful, resulting in clinical and radiographic resolution. It is recommended that Cryptococcus neoformans be considered in the differential diagnosis of community acquired pneumonia, even in immunocompetent patients.

Cryptococcus neoformans is a ubiquitous organism and is recognized as a cause of human infection worldwide. It has five serological serotypes (A, B, C, D and AD) and two mycological classes (Cryptococcus neoformans var neoformans and Cryptococcus neoformans var gattii.)

The lungs, where cryptococcus multiplies by binary fission in the alveoli, are the most common portal of entry that results in human infection. The large polysaccharide capsule that surrounds the organism is an antiphagocytic virulence factor. $\mathrm{C}$ neoformans produces no identified toxins and rarely causes tissue destruction. When tissue damage occurs, it usually does so outside of the lung, most commonly when dissemination to the central nervous system occurs. The pathogenesis of tissue injury relates to the inflammatory reaction that $\mathrm{C}$ neoformans induces.

Diagnosis can be made by the detection of cryptococcal antigens in the serum, cerebrospinal fluid or other infected body fluid by means of a latex agglutination assay, which reacts in the presence of the polysaccharide capsule antigen. This test has been highly reliable for the diagnosis of cryptococcal infection (1).

Cryptococcal infection is increasing in incidence, primarily due to the HIV epidemic. It is usually seen in the context of advanced HIV disease (CD4-lymphocyte counts of less than $100 / \mathrm{mm}^{3}$ ) (2). Although cryptococcal infection presents most commonly as meningitis, pulmonary involvement occurs in $25 \%$ to $50 \%$ of HIV-positive patients $(2,3)$. The pathogenesis of pulmonary cryptococcosis in immuno- competent patients is not well known (1). Important host defence mechanisms against cryptococcal infection include $\mathrm{T}$ lymphocytes and cell-mediated immunity. It is, therefore, not surprising that patients with $\mathrm{T}$ cell immunodeficiency, secondary to immunosuppressive therapy, corticosteroids and adult $\mathrm{T}$ cell leukemia are at an increased risk of infection (4).

Due to the ubiquitous nature of Cryptococcus neoformans, tissue invasion, rather than culture from sputum, is necessary for the diagnosis of pneumonia (5). Once diagnosed, there is no consensus on the ideal treatment of pulmonary cryptococcosis in the immunocompetent host. Aberg et al (6) suggested that no antifungal therapy is required; however, this was based on a small case series and was suggested before the widespread availability of azole derivatives such as fluconazole (7), which carry considerably less risk of toxicity than do agents such as parenteral amphotericin B. Yamaguchi et al (8) reported a nearly 90\% clinical response rate to fluconazole in patients with documented cryptococcoccosis who do not have AIDS.

Traditionally, cryptococcosis in HIV-positive patients was treated with amphotericin B, but evidence now supports the use of azole derivatives. For initial therapy,
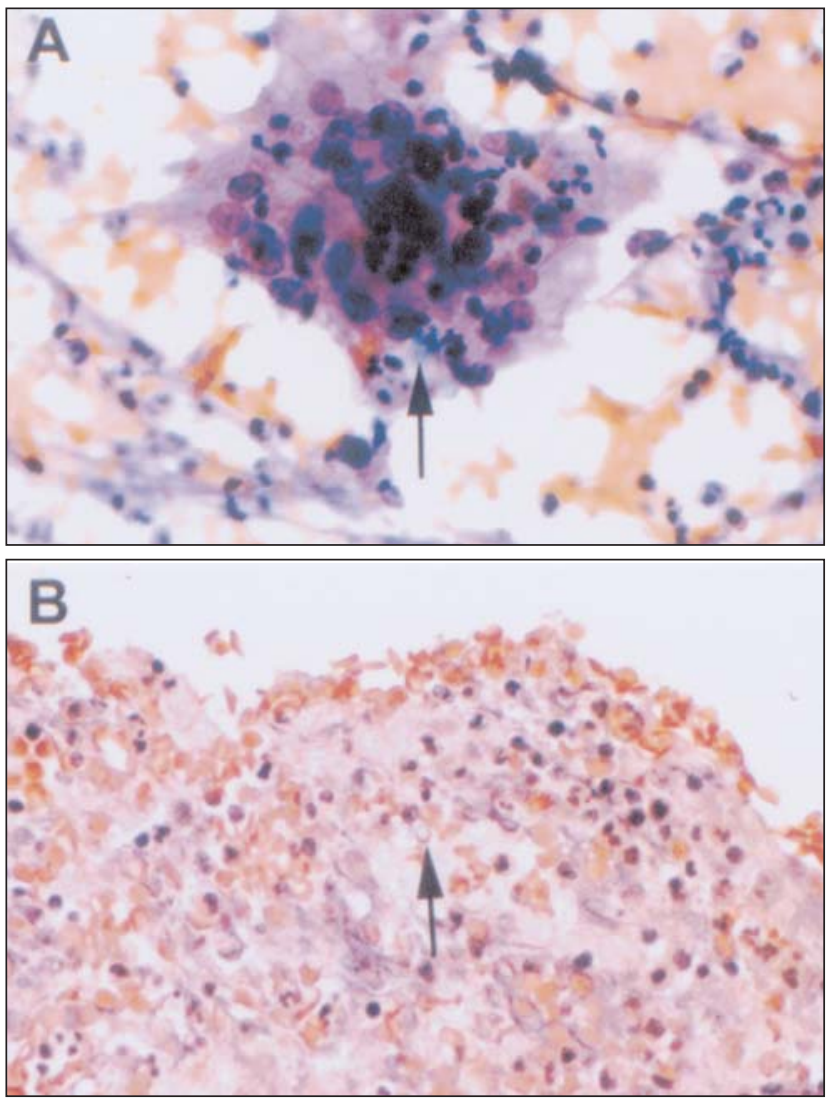

Figure 2) A Before therapy, a lung biopsy demonstrated Cryptococcus species in blue (arrow). (PAP stain, original magnification $\times 60$ ). B Before therapy, lung biopsy demonstrated Cryptococcus neoformans capsule in dark pink (arrow). (Mucin stain, original magnification $\times 60$ ) 


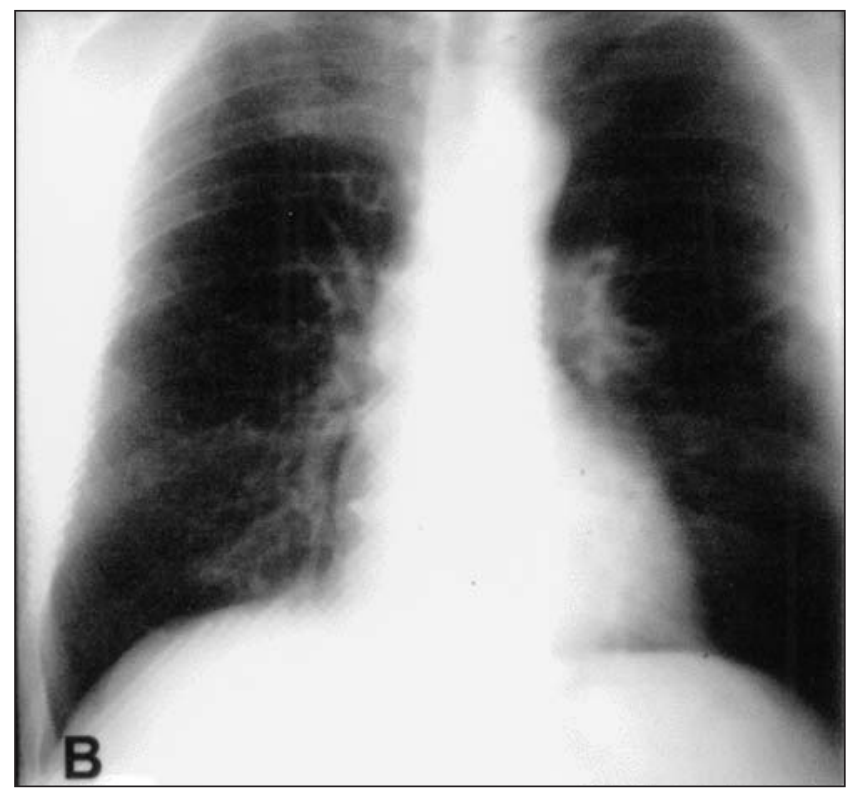

Figure 3) Chest radiography after completion of therapy (six months later). X-ray reveals a resolution of the infiltrate with minimal scarring

Meyohas et al (9) demonstrated a favourable response in six of 11 patients with pulmonary cryptococcosis who were treated with fluconazole. Saag et al (10) demonstrated a similar response rate in $194 \mathrm{HIV}$-positive patients with cryptococcal meningitis who took fluconazole compared with amphotericin B. Furthermore, fluconazole has been shown to be safe and effective for maintenance therapy of cryptococcal meningitis in patients with HIV infection (11). No data suggest that maintenance therapy is required or is necessary in immunocompetent hosts.
We report a case of pulmonary cryptococcosis in an immunocompetent patient. Fluconazole therapy led to both clinical and radiographic resolution of the disease. Cryptococcus neoformans should be considered in the differential diagnosis of pneumonia - even in immunocompetent patients.

\section{REFERENCES}

1. Saag MS, Graybill RJ, Larsen RA, et al. Practice guidelines for the management of cryptococcal disease. Infectious Diseases Society of America. Clin Infect Dis 2000;30:710-8.

2. Gal AA, Koss MN, Hawkins J, Evans S, Einstein H. The pathology of pulmonary cryptococcal infections in the acquired immunodeficiency syndrome. Arch Pathol Lab Med 1986;110:502-7.

3. Chechani V, Kamholz SL. Pulmonary manifestations of disseminated cryptococcosis in patients with AIDS. Chest 1990;98:1060-6.

4. Mody CH, Warren PW. Host defences to pulmonary mycosis. Can J Infect Dis 1999;10:147-55.

5. Jensen WA, Rose RM, Hammer SM, Karchmer AW. Serologic diagnosis of focal pneumonia caused by Cryptococcus neoformans. Am Rev Respir Dis 1985;132:189-91.

6. Aberg JA, Mundy LM, Powderly WG. Pulmonary cryptococcosis in patients without HIV infection. Chest 1999;115:734-40.

7. Sarosi GA. Cryptococcal lung disease in patients without HIV infection. Chest 1999;115:610-1.

8. Yamaguchi H, Ikemoto H, Watanabe K, Ito A, Hara K, Kohno S. Fluconazole monotherapy for cryptococcosis in non-AIDS patients. Eur J Clin Microbiol Infect Dis 1996;15:787-92.

9. Meyohas MC, Roux P, Bollens D, et al. Pulmonary cryptococcosis: localized and disseminated infections in 27 patients with AIDS. Clin Infect Dis 1995;21:628-33.

10. Saag MS, Powderly WG, Cloud GA, et al. Comparison of amphotericin B with fluconazole in the treatment of acute AIDS-associated cryptococcal meningitis. The NIAID Mycoses Study Group and the AIDS Clinical Trials Group. N Engl J Med 1992;326:83-9.

11. Powderly WG, Saag MS, Cloud GA, et al. A controlled trial of fluconazole or amphotericin B to prevent relapse of cryptococcal meningitis in patients with the acquired immunodeficiency syndrome. The NIAID AIDS Clinical Trials Group and Mycoses Study Group. N Engl J Med 1992;326:793-8. 


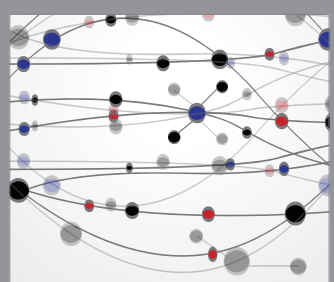

The Scientific World Journal
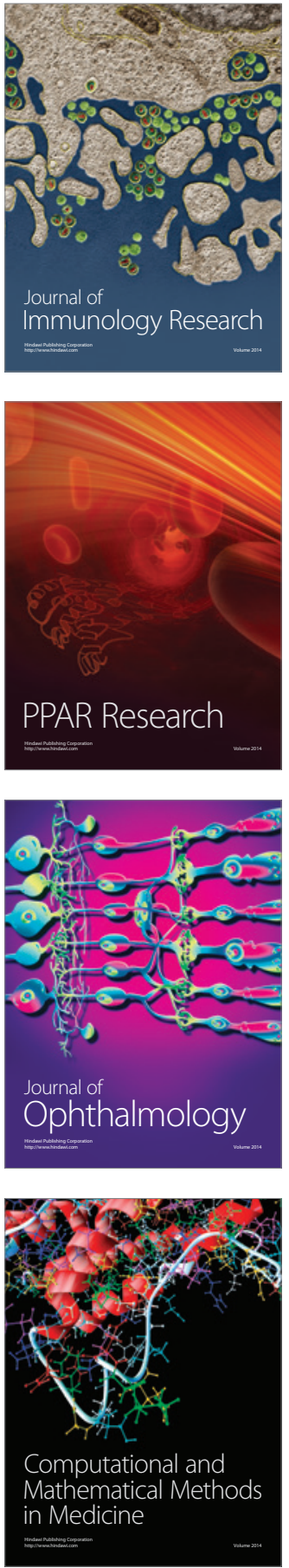

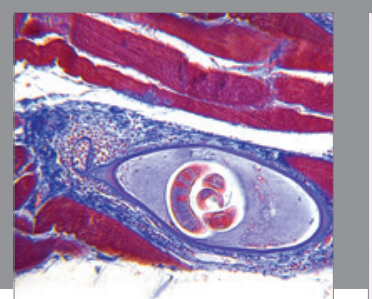

Gastroenterology Research and Practice

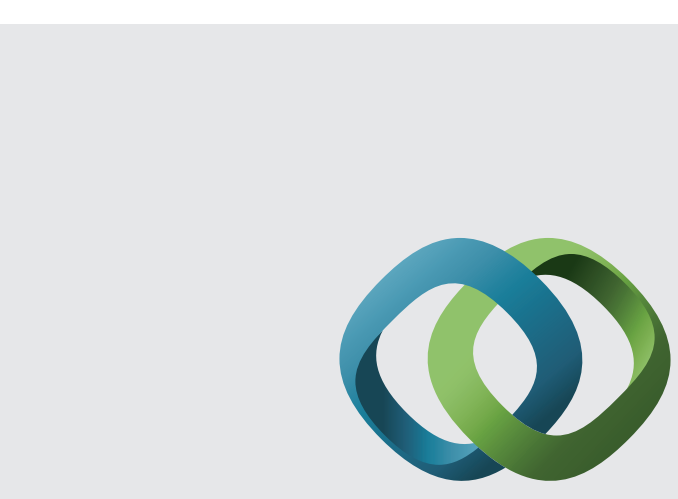

\section{Hindawi}

Submit your manuscripts at

http://www.hindawi.com
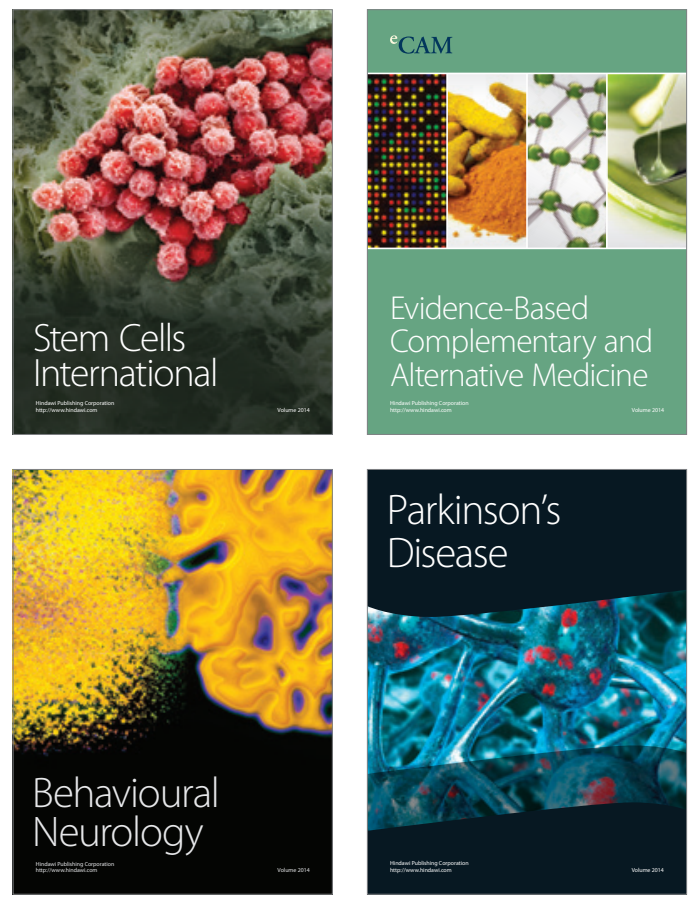
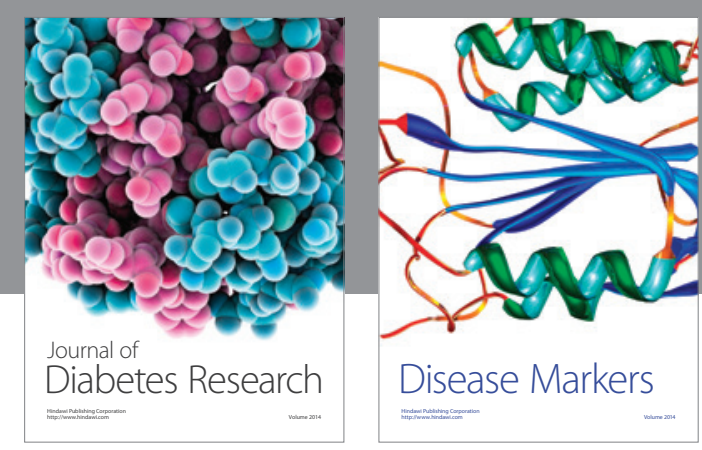

Disease Markers
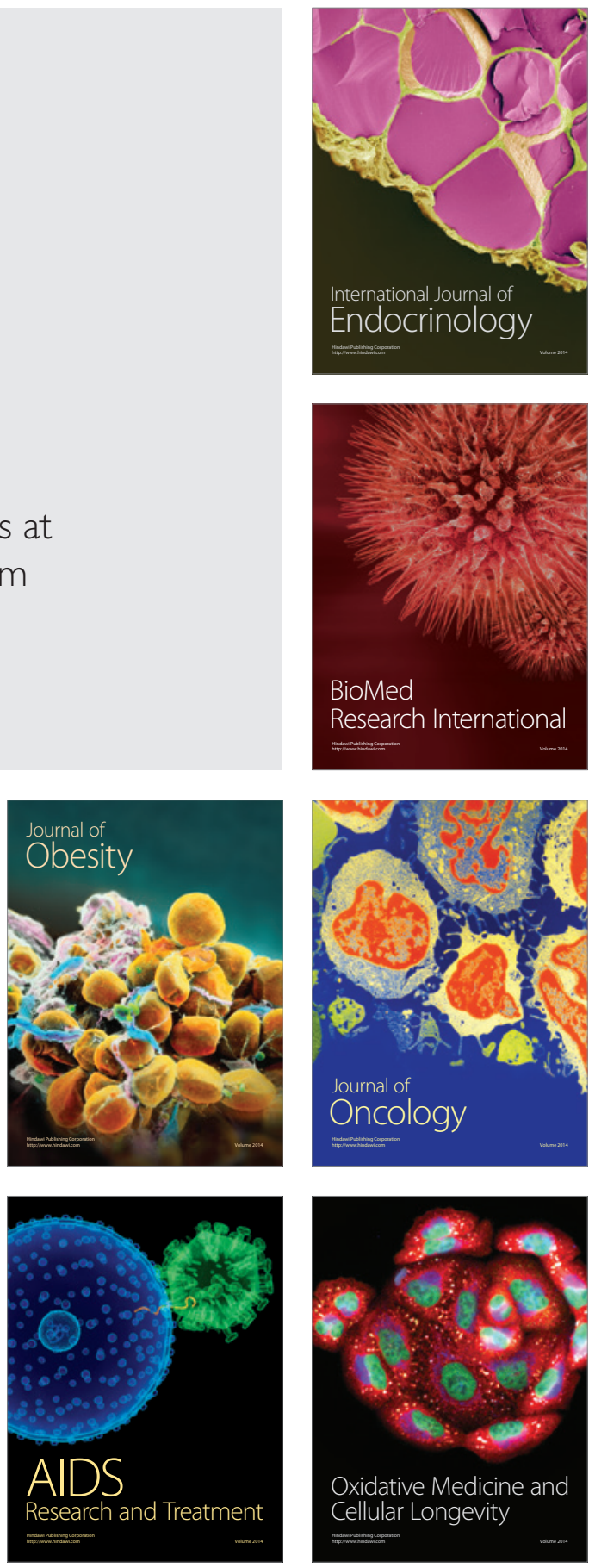